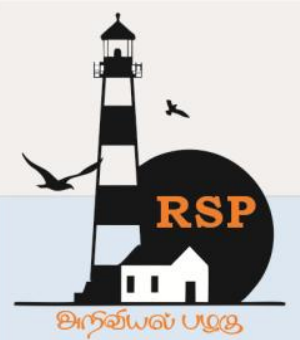

INTERNATIONAL RESEARCH JOURNAL ON

e-ISSN : 2582 - 4376 ADVANCED SCIENCE HUB Open Access

RSP SCIENCE HUB

(The Hub of Research Ideas)

Available online at www.rspsciencehub.com

\title{
Machine Learning: An Intuitive Approach In Healthcare
}

Dr. Salini Suresh ${ }^{1}$, Prof. Suneetha. ${ }^{2}$, Ms. Niharika Sinha ${ }^{3}$, Mr. Sabyasachi Prusty ${ }^{4}$, Mr. Sriranga H. $A^{5}$ 1,2,3,4,5 Dayananda Sagar College, Bangalore, Karnataka.

salinisuresh-bu@dayanandasagar.edu1,hod-mcabu@dayanandasagar.edu², niharikasinha3011@gmail.com ${ }^{3}$, sabyasachiprusty@gmail.com ${ }^{4}$,sriranga.ha98@gmail.com ${ }^{5}$

Abstract

Health is a crucial resource for a person's being to measure in our society from any disease. The fast development of the population appears to be trying to record and dissect the massive measure of knowledge about patients. Healthcare may be a need, and clinical specialists are constantly attempting to get approaches to actualize innovations and give effective outcomes. The main problem faced by the healthcare industry is the rising costs which include diagnosis and prediction of diseases, drug discovery, medical imaging diagnosis, personalized medicine, behavior therapy, and smart health records. Machine learning gives us an advantage of processing these information naturally which helps in making the human services framework progressively powerful. Getting the correct determination may be a key part of Healthcare. It clarifies a patient's medical issue and suggests health care treatment. The disease diagnostic technique is a complex, community-oriented action that has clinical, intelligent and data social events to make a decision about a patient's medical issue. Google has built up a ML model to assist recognize dangerous tumours on mammograms. Stanford's profound learning calculation to differentiate skin malignancy. This paper is focused on the importance of Machine Learning in Healthcare just like the different application areas, latest research works in healthcare, wise machine learning contribution in Healthcare, and so on. Machine Learning is an application of Artificial Intelligence that helps in automatically learning and improving itself from experience. It is used in many other sectors like Law, Marketing \& Advertising, Finance, Retail\& Customer Services and Healthcare which also includes Covid-19. This paper presents various research in the Medicine and Healthcare sector

Keywords: Healthcare, Machine Learning, Artificial Intelligence, Predict and Diagnosis of Disease COVID, Pattern Recognition, Decision Making.

\section{Introduction}

The definition of health, it's a normal state of a human is free of physical, mental and social illness. How we can say that a human has good health, they have good relationships, environment, and education. As a human, genetics behavior is an important factor. Good health doesn't mean the absence of disease; it's a kind of ability to recover from the illness that affects humans and quick relief from disease. There are good practices like healthful diet, daily exercise, regular check-up, and coping approaches that help us to prevent or quickly recover from diseases. Living with good health in our daily life, we can avoid and easily recover chronic diseases and long-term illnesses. You are living in a healthy condition by taking care of a healthy lifestyle. WHO said that good health is central to human contentment? It will make a good contribution to our economic betterment. A well-being human can work more productively and live longer. Improvement of the country's people's health government is also 


\section{www.rspsciencehub.com}

more concerned and supporting. The economic times of India reported the rising cost of medical treatment nearly $50 \%$ within 5 years. As compared with past decades, advancement in medical innovation has made it conceivable to cure diseases that were once viewed as serious. In any case, the expense of their treatment is high, and it is practically unimaginable for a middleclass individual to bear the cost of them. This makes it certain that despite the fact that human healthcare services have advanced colossally, their reasonableness keeps on staying distant for most Indians.

From drugs to medical procedures, to other clinical strategies, the costs are exactly having a couple of lakhs. Shows the report of the selection of digital Healthcare in the country. Prato Blog demonstrates that India starts to gain endorsement in 'Digital Healthcare'. Indian people consulted their doctors 3.2 times in 2018 as we compared with 2017 its 2.7 times. In the future also, this number is going up. The healthcare area creates roughly one trillion gigabytes of medical information annually. An expansion has joined these massive amounts of information in modest, enormous scope processing power. Coming together, they increase the chances that improves the disclosure of new therapeutics and to make the movement of current ones dynamically reasonable. Regardless of the advancements in improvement of medicals, treatment recommendations and diagnostics there have been mistakes and propositions that ML has shown up at top hype. Some investigation is legitimized, yet AI continues offering the transformative potential for prosperity and restorative administrations.

Medicine has lots of manual processes. Lab values, diagnoses and other chart notes are handwritten on paper. This is an area where technology helps to improve the workflow which also helps to improve the patient care. Machine learning provides such a platform where it helps the doctors analyse a patient in an easier way. It has virtually endless applications in the healthcare industry like helping to streamline administrative processes in hospitals and it personalises medical treatments.
An algorithm has been developed using ML by Google which helps in identifying cancerous tumours on Mammograms. By optimizing the way the systems are designed, Quotient Health developed a software using ML which targets to reduce the cost of EMR (Electronic Medical Records) systems. This is more cost-efficient.

Doctors and physicians can predict the health risk of a population by identifying patterns by using ML. It surfaces high risk markers, model disease progression, etc. ML is also used to exchange health information, clinical data and improves the workflow which helps in modernizing workflows in the health care sector. Clinical records of millions of patients are being analysed everyday using ML which helps in early detection of diseases, therapy requirements are pinpointed and clinical trial opportunities are highlighted.

The difference between a healthy anatomy and a tumour is found out using ML from 3D radiological images. This project called the Inner Eye is conducted by Microsoft. This helps the medical experts in radiotherapy and planning surgeries. IBM's Watson AI technology with ML is used in immuno-oncology research. This research is being done to learn how the body's immune system fights cancer. ML is also used in the Interrogative Biology platform for mapping diseases and oncology, neurology and other rare condition treatments. By these, health care providers take more of a predictive approach towards the treatment rather than taking a trialand-error approach. [1-4]

\section{What is Machine Learning?}

The study of algorithms improving on its own through its experience is known as Machine Learning. It is a division of Artificial Intelligence. Based on some sample data, a mathematical model known as training data is built by the ML algorithms which helps in deciding or making predictions without being clearly programmed to do so. ML algorithms are used in various applications like computer vision, filtering emails where it is tough to develop general algorithms to perform the required tasks. 
ML in Healthcare brings two sorts of areas: software engineering and clinical science in a common string. Machine learning can be trained to look at medical data, distinguish anomalies, and point to zones that need consideration, subsequently improving the precision of all these processes. ML can offer a target assessment to improve productivity, unwavering quality, and exactness.[5-8]

There are three traditional approaches to machine learning:

1. Supervised Learning: In this a sample input and a needed output is provided by a teacher as an example in order to learn a general rule that maps inputs to outputs.

2.Unsupervised Learning: In this learning algorithms are not provided with labels so it finds structure on its own in its inputs.

3. Reinforcement Learning: In this a computer program performs a certain goal by interacting with a dynamic environment.

\subsection{Machine Learning Applications In Healthcare}

ML in healthcare services assists with investigating a large number of various information focuses and recommends results, gives auspicious risk scores, exact asset allotment, and has numerous different applications. The attention on the best way to utilize ML to expand persistent consideration. For instance, on the off chance that specialists are testing a patient for cancer, at that point they need the most excellent biopsy results to determine. ML calculation that can audit the pathology data and help the pathologist with a determination is important. In the event that specialists can get the outcomes in a small amount of the time with an indistinguishable level of precision, at that point, at last, this will improve tolerant consideration and fulfillment.

\subsubsection{Identifying Diseases and Diagnosis}

One major application of ML in the Healthcare sector is to identify and diagnose diseases which are or else very difficult to be diagnosed. Diseases like cancer and other genetic diseases which are very difficult to catch in the initial stage can be included in it. One such example is IBM Watson Genomics which integrates cognitive computing with genome-based tumour sequencing to improve the speed of diagnosis. Therapeutic treatments using AI in areas like oncology are being developed by Berg, a biopharma giant. A commercially feasible solution is being developed by P1vital's Predict (Predicting Response to Depression Treatment) for diagnosis and treatments in routine clinical conditions.

\subsubsection{Drug Discovery and Manufacturing}

One primary application of ML in the healthcare sector is in the early stage of the drug discovery process. $R \& D$ technologies like precision medicine and next-generation sequencing are some of the things which can alternatively find paths for therapy of multifactorial diseases. Presently the ML techniques use unsupervised learning which finds patterns in the data without any given instructions. One such project has been developed by the tech-giant Microsoft using ML. This project Hanover uses AI based technology for the treatment of cancer and personalized drug combinations for AML (Acute Myeloid Leukaemia).[9-11]

\subsubsection{Medical Imaging Diagnosis}

Computer Vision is one breakthrough technology created using Machine Learning and Deep Learning. This has also been accepted by the project Inner Eye, an initiative by Microsoft which works on diagnostic tools for image analysis. As machine learning becomes more popular with time it is expected to see more sources of data from different medical imagery in becoming a part of this AI-driven diagnostic process.

\subsubsection{Personalized Medicine}

By pairing an individual's health with predictive analytics, treatments that are personalized can not only be more effective but can also be used for further research and better assessment of diseases. Presently, doctors or physicians only have specific sets of diagnoses or the risk estimation of patients based on the history of their symptoms and their genetic information. ML in medicine is showing great promise, one of the forefront movements being by IBM Watson Oncology which helps in generating multiple treatment options. In the near future, we are going to witness even more developed devices and biosensors with health 
measurement capabilities coming on the market, allowing the collection of even more data's for healthcare related technologies.

\subsubsection{Machine Learning-based Behavioral Modification}

Modification in behavior is a very important part of preventive medicine, and ever since the entrance of ML in the healthcare sector, many start-ups are researching in the cancer prevention and identification, treatment of patients field etc. A company named Somatic which is a B2B2Cbased data Analytics Company has released a Machine Learning based app for gesture recognition that we make in our daily lives which allows us to better understand our unconscious or subconscious behavior and make the needed changes.

\subsubsection{Smart Health Records}

Up-to-date maintenance of health records is a very exhaustive process. While technology has eased the process of data entry, the fact is that a majority of processes still takes a huge amount of time to complete. The most important role of ML in the healthcare sector is to reduce the amount of time, effort and money. Methods that classify documents using vector machines and ML based OCR recognition techniques are slowly gathering steam. Technologies like Google's Cloud Vision API and MATLAB's machine learning-based handwriting recognition. MIT today is at the verge of developing a next generation technology of intelligent and smart health records, which will use ML-based tolls from the base with diagnosis and suggestions in clinical treatments, etc.

\subsubsection{Clinical Trial and Research}

In the field of clinical research and trials, ML has various capable applications. As seen in healthcare sectors, some of the clinical trials cost a lot of time and money. Many cases can take even years to complete. ML based predictive analytics applied to identify potential clinical trial candidates can help developers in drawing a wide variety of data points such as previous clinical records ,visits ,treatments, social media and etc. Machine Learning can also monitor in real time and access data of the trial participants which can help in finding the best sample size to be tested and data based errors can be reduced by leveraging the power of electronic records.

\subsubsection{Crowdsourced Data Collection}

Nowadays in the medical field Crowdsourcing is at rage which allows researchers and doctors to have access to a wide variety and amount of information uploaded by the public with their personal consent. In the way medicine will be perceived in the future, this live health data has great ramifications. ML based facial recognition is applied by Apple's research kit which allows users to access apps that are interactive to try and treat Asperger's and Parkinson's disease. Based on the Crowdsource information to decipher, accumulate, and make available diabetes and insulin data in real time IBM recently partnered with Medtronic. With the technological advancements in Iota, new ways are being discovered by healthcare industries to use these data and tackle the cases that are very tough to diagnose which would help in the overall improvement of medication and diagnosis.

\subsubsection{Better Radiotherapy}

Radiology is one of the most sought-after applications of ML in healthcare. There are many discrete variables in Medical image analysis which can arise at any moment or any particular time. Using complex equations there are many lesions, cancer foci, etc. that cannot be simplified. Since algorithms that are based on Machine Learning adapt and learn from various samples on hand, it becomes very easy to diagnose and find the variables. One very popular use of Machine Learning in medical image analysis is classifying lesions into categories such as normal or abnormal., lesion or non-lesion ,etc. Researchers are actively being helped by Google's DeepMind Health in UCLH to develop algorithms which help in detecting the difference between healthy and cancerous tissues and improve radiation treatment for it.

\subsubsection{Outbreak Prediction}

Today, in monitoring and predicting epidemics around the world AI-based technologies and machine learning is being used. From the satellites, real time social media updates, website information, etc. scientists have access to huge amounts of data. Artificial neural networks collect these information and predict everything starting from malaria outbreaks to severe chronic infectious diseases. Predictions of these outbreaks are especially helpful in third-world countries as they lack any crucial educational system and crucial medical infrastructure. One primary 
example of this is the Pro-MED-mail which is an internal-bases reporting platform. This platform monitors diseases that are evolving and emerging and provide outbreak reports in real-time.

\subsection{Projects and Devices Using Machine Learning In Healthcare \\ 2.2.1 InnerEye:}

This is a project by Microsoft. Tools are made using ML to automatically and quantitatively analyse 3-D radiological images. Further this project turns these images to measuring devices.

ML techniques are developed by project InnerEye for the automatic description of tumors and healthy anatomy in three dimensional radiological images.

The InnerEye technology may enable:

1. Extraction of targeted radiomics measurements for quantitative radiology,

2. Efficient contouring for radiotherapy planning

3. Precise surgery planning and navigation.

Multi-dimensional radiological images are turned into measuring devices using project InnerEye in practice.

Many years of research in ML and Computer Vision built the project InnerEye. Algorithms like Deep Decision Forest which has been already used by Kinect and Hololens and Convolutional Neural Networks which are used for the automatic, voxelwise segmentation of medical images have been employed by it.

Designing of this technology is being done under the guidance of physicians and surgeons. Expert clinical researchers can refine and adjust the results of the ML operations until they are totally satisfied with the results. They are under the complete control of the result all the time.

\subsubsection{IBM Watson Oncology:}

Leading Oncologists' deep expertise in cancer care is combined with the speed of IBM Watson by Watson for Oncology to help physicians as they consider personalized potential cancer treatment for their patients.

Watson for Oncology is a solution that has all the information from required guidelines, medical journals, best practices and textbooks, etc. The solution analyses information from a patient's medical history and treatment records and displays possible treatment options ranked by success rates or level of confidence based on the evidence from previous treatment records. The oncologists then apply their expertise on the field and decide on the most appropriate treatment for the patient

\subsubsection{Verily by Alphabet Inc.}

Preventive care can help people to live a happy and healthy life. It can also lower the cost of healthcare for the entire population. Verily aims to perpetuate a stronger and a better feedback loop which supports the learning health system where with better data and access to the research and care, interventions and care can continuously improve.

Verily believes that the change must happen from the inside. So they are forging a collaboration across the entire healthcare community and ecosystem, from life sciences companies to hospitals to academic research institutions to health systems.

A visible impact on human health can happen by likeminded industry experts joining forces with each other. Verity's partnership with various industries and companies can create a solution that can significantly have a positive impact on health outcomes on a huge scale. By putting significant investment in both basic science and big public health initiatives they are working relentlessly to develop a next generation healthcare innovation. And by collaborating with world renowned organisations the company is committed in making a positive impact on human health.

\subsubsection{Ondo:}

It's a project based on machine learning by Verily which comes under Google's parent company Alphabet Inc. that aims to help people that have type 2 diabetes to live a full and healthy life. It is developing a comprehensive solution by combining softwares, devices, medicines and professional care for diseases to be managed simply and in a more intelligent way.

It is a virtual care program with clinical support, diabetes tools and coaching for the people suffering from type 2 diabetes to take control of it. The users of Onduo download the Onduo app which provides them with a blood glucose monitor(BGM) and in few cases a continuous glucose monitor(CGM) to get insights on their daily habits and their decisions have an impact on their health and level of glucose. The users also have an access to the app's care team which is 
made up of diabetes professionals like diabetes educators, dieticians and physicians who provide personalized guidance and help regarding the activities, lifestyle habits, medication management and diet of the affected.

Data from the initial users of the Onduo app suggests that participation in this program helped in significant improvement in haemoglobin A1C, which is an indicator of the disease with up to six months of follow up with the ones who were previously not meeting with their treatment targets.

\subsubsection{Smartwatches:}

Wearing a smartwatch or a wearable is just not a fashion statement anymore. These devices are increasingly providing healthcare industries and experts with potential information about a patient between appointments or after surgery which helps in the better treatment of the person.

An average of more than 150 million smart wearables are being sold every year with an annual growth of $15.3 \%$. According to IDC research, the majority of that boost is coming because of the adoption of healthcare segments in the smartwatches.

Even though the numbers include smart clothing, step counting shoes, wristbands,etc., nearly half of all sales are of the smartwatches as cited in the research. This could be due to the Food and Drug Administration giving clearance to the Apple Watch for its electrocardiogram and irregular heart rhythm monitoring functions.

Loads of data collected properly giving respect to an individual's privacy has the potential to decrease healthcare cost by reducing the number of times an individual takes medical visits and by detecting potential threats in its infancy by records collected by the smart wearable.

\subsubsection{Apple Watch Helps Collect Heart Health Data}

Ochsner Health System in New Orleans which launched a pilot program to better track patients with uncontrolled hypertension in 2015 has been inspired by the Apple Watch. Apple watch is the first of its kind which measures a patient's blood pressure just by wearing it and wirelessly records the data which helps the patient in managing a chronic condition.

Apple watch is now also being used by the organization to monitor a patient with atrial fibrillation and it has outfitted doctors too. Dr. Richard Milani,Chief clinical transformation officer of Oschner recently told Health Tech that alerts regarding a patient's declining condition can now be sent directly to a provider's wrist even if they are wearing gloves.

However Ochsner is not the only hospital doing it. 47 percent of hospitals are providing their patients who have chronic diseases with smart wearables as for a survey of hospital executives from the Management Systems Society, Healthcare Information and AT\&T. A third of the hospitals issue the devices to preventive care and post-op.

\subsubsection{Smartwatch Data Boosts Medical Studies and Patient Care}

Given the advanced functions of these watches ,they are also a boon to the researchers. Stanford University this year announced the results of a study which found that Apple Watch with $84 \%$ accuracy could detect atrial fibrillation which is a leading stroke risk. Exciting and rapid developments are being made to increase the analytical power of these tools.

Apple made the headlines in September after announcing three medical studies that involved institutions such as Harvard University and the World Health Organisation. This partnership will collect data generated from the users of the Apple Watches and the company's research app with women's menstruation and overall health, everyday noise exposure and hearing loss and the relationship between movement and heart health being in focus.

Even other manufacturers are embracing data-driven wellness functions which can help patients and the providers to keep tabs. Now a user can track the wearer's blood pressure by Samsung's Galaxy Watch and Fitbit has advanced from just being an activity tracker and has partnered with Google Cloud which can help the users in safely transmitting the health data to their doctors and their electronic medical records.

\section{A Look towards Challenges}

\subsection{Data Quality}

Healthcare data vary significantly in completeness, consistency, accuracy. These differences arise from incentives that drive healthcare providers, the extent of coaching they receive, the culture and 
complexity of various institutions or places of service. As a result, errors and gaps are common.

Lots of data cleaning (often manual) has got to happen before any proper analysis are often done. As such, ML that automatically ingests, processes data would inherit a lot of these issues of data quality, which makes the models less sensitive to "true" patterns, and are often led down spurious, anomalous paths.

\subsection{Working with Multiple data Sets}

Healthcare data traditionally exists in different silos. While some commonality exists, different sources usually have materially different data structures. Any analytic effort requires gathering, restructuring, and mixing various datasets. This process is more often complex, manual and subject to vary .Whenever institutions change IT systems, enormous challenges occur in stitching together patient data over time. All these issues make automated ML processes difficult. Such deviations in arrangement also make scaling up the deployment of ML algorithms challenging.

\subsection{Medical Complexity}

Medicine is complex. From physicians, to various treatment modalities, to thousands of diseases, to clinical judgments, to regional practice differences, there are often endless permutations of the profile of patients and repair delivery. ML algorithms can be difficult on meaningful patterns given these complexities.

\subsection{Consistency over Time}

One of the major tenets of $\mathrm{ML}$ is that the algorithms can recalibrate more data arrival. While this is a strength, the dynamic nature of the patients profiling can make it too difficult to compare clinical outcomes. For example, if we have to identify patients to gauge whether a specific drug is helpful in addressing a medical condition, akin to how clinical trials work. If the patient profiling algorithm changes over time, the comparison between controls versus intervention groups may become inconsistent.

\subsection{Deployment Challenges}

Assuming to manage the ML methodology and refine so that it can arrive at an effective application which may result in, that often the recommendations may not be as well received by doctors as you anticipated. For various reasons, the doctors may not find the results useful. There may be aspects of the patient that the ML algorithm could not detect. The doctors may not care about another practice alert.

\subsection{Time and Capital Investment}

ML has very high efficacy in specific use cases, like imaging processing, where the input file is usually uniform, and therefore the traditional human workflow has been manual. The wider use of applications using ML have had limited success, making justification of the time, resource investment tenuous.

\section{Proposed System}

However, what's immediately plausible, and will therefore be planned for, may be a federation of 'narrow and 'targeted' machine learning systems that are ready to handle core information science problems across health system by extending capabilities of human decision makers, and in so establishing new standards of functional and efficient enough in clinical and management operations. This is a big opportunity for health system transformation because the cost of augmenting decision-making capabilities across the health system is unlikely to be large. There is no other approach that gives such potential impact without commensurate scaling of cost. The fixed charge involved in developing machine learning solutions: the cost of research and development and of retooling a health system is considerable, but given the potential scalability, the rationale to take a position is obvious .An opportunity exists to seed growth in machine learning through the creation of high resolution clinical data sets and therefore the necessary mechanisms for sharing of knowledge and collaborative investigation to determine both efficacy and safety. What is currently missing in health systems is the leadership to try to do so. Whilst the issues raised are being actively discussed among the tutorial AI community, the tutorial AI community alone won't be ready to solve them; it'll require leadership from policy makers and the engagement of citizens, patients and clinicians. The agitation of wholesale displacement of health workforce by $\mathrm{AI}$ is overstated, but where fear is warranted is in considering the opportunity cost of not embracing AI, of continuing business as was common with piecemeal implementation of AI that doesn't realize its potential for transformation of health systems. 


\section{Conclusion}

Artificial Intelligence, Machine Learning, and Deep Learning have increased a great deal of consideration for a long while now. Machine learning assembles a gigantic volume of information, including patients' records, clinical reports, and protection records, and applies its models to give the best results. All the more significantly, researchers and scientists are utilizing ML to produce various brilliant arrangements that can eventually help in diagnosing and treating an ailment. ML is able to do all the more precisely identifying an illness at a previous stage, assisting with lessening the number of readmissions in medical clinics and centres. The inexorably developing number of uses of Machine Learning in medicinal services permits us to look at a future where information, investigation, and advancement work connected at the hip to help incalculable patients without them consistently acknowledging it. In the above chapters, we discussed what the roles ML plays in Healthcare are. Before long, it will be very normal to discover ML-based applications installed with constant patient information accessible from various social insurance frameworks in different nations, in this manner, expanding the viability of new treatment choices that were inaccessible previously.

\section{Future Scope}

The current study shows the contribution of machine learning in Healthcare. Machine Learning is growing technology, and it can quickly converge with other technologies like block chain, Internet of Things (Iota). The advancement of these technologies will help to address the critical challenges of Healthcare in the future.

\section{References}
[1]. https://builtin.com/artificial- intelligence/machine-learning- healthcare
[2]. https://www.microsoft.com/en- us/research/project/medical-image- analysis/
[3]. https://www.flatworldsolutions.com/he althcare/articles/top-10-applications-of- machine-learning-in-healthcare.php

[4]. https://verily.com/stories/intelligentdisease-management/

[5]. https://healthtechmagazine.net/article/2 019/10/smartwatches-healthcare-driveinsights-and-

action\#: :text=Smartwatch\%20Data $\% 2$ 0Boosts\%20Medical\%20Studies\%20an $\mathrm{d} \% 20$ Patient $\% 20$ Care \&text $=$ This $\% 20 \mathrm{y}$ ear $\% 2 \mathrm{C} \% 20$ Stanford $\% 20$ University $\% 2$ 0announced,risk \%2C\%20with\%2084\% 20percent $\% 20$ accuracy.\&text=Samsun g's\%20Galaxy\%20Watch\%20can\%20n ow\%20track\%20a\%20wearer's \%20blo od\%20pressure.

[6]. Singh, P., Singh, S., and Panda-Jain, G. S. (2018).Effective heart disease prediction systemusing data mining techniques. International Journal of Nanomedicine

[7]. Mohan, S., Tirumala, C., and Srivastava, G. (2019). Effective Heart Disease Prediction using Hybrid Machine Learning Techniques, IEEE

[8]. Coffin, R., Travaglioni, M., Piscitelli, G., Petrillo, A. \& De Felice, F. (2020). Artificial Intelligence and Machine Learning Applications in Smart Production: Progress, Trends, and Directions. Sustainability

[9]. Discon, R.F, Zisser, H., \& Layne J.E., et al (2019). A Virtual Type 2 Diabetes Clinic Using Continuous Glucose Monitoring and Endocrinology Visits. Journal of Diabetes Science and Technology.

[10]. Francesca Bonin (2018). Could AI Help People Change Their Behaviour IBM Blog?

[11]. PRACTO. (2019). India is getting serious about Healthcare: Practo Insights. Practo Blog 